\title{
CHECKLIST OF CHAMPION TREES IN THE CZECH REPUBLIC
}

\author{
LUBOŠ ÚRADNÍČEK, MARTIN ŠRÁMEK, JAROMÍRA DRESLEROVÁ
}

\begin{abstract}
Department of Forest Botany, Dendrology and Geobiocoenology, Faculty of Forestry and Wood Technology, Mendel University in Brno, Zemédělská 3, 613 00, Czech Republic, e-mail: lubos.uradnicek@mendelu.cz
\end{abstract}

Received: $7^{\text {th }}$ September 2017, Accepted: $28^{\text {th }}$ September 2017

\begin{abstract}
An increasing number of people all around the world have become interested in remarkable and magnificent trees in recent years. Accessible databases of champion trees are available. Unfortunately, the Czech Republic does not have a single database of remarkable or champion trees. We have old tradition to find and describe an old and monumental trees in our country. In 1899 the first book was issued about it. Many of the biggest specimens are already registered in the Monumental Trees database, which is run by the Nature Conservation Agency of the Czech Republic, registered a total of 25,598 memorable trees in 2017. Not all remarkable or champion trees are designated as memorable. Due too we present the known data on champion trees in an independent checklist. There are shown 116 species of trees with their girth and other information as height, GPS coordinate etc. from various sources. This checklist is a basis for a future one national database. When comparing the champion trees of the Czech Republic with the databases of other European states, particularly that of Monumental Trees, it emerges that we are not far from coming first. The Czech champion tree, Vejda's Lime Tree in Pastviny (Tilia platyphyllos Scop.) measured $1,305 \mathrm{~cm}$ at girth in 2015. We would like to prepare one integrated Czech database of remarkable trees and champion trees by help of the Professor August Bayer Foundation as soon as possible.
\end{abstract}

Keywords: remarkable trees, databases trees, champion trees, monumental trees

\section{INTRODUCTION}

Trees are an integral part of both nature and the cultural environment of people. Some of them grow to magnificent sizes, others only several meters in height, the conditioning factors being the respective species, age and growth conditions.

Magnificent trees were worshipped already in ancient times by our ancestors who believed that trees house supernatural beings. In modern times locating magnificent trees has become a hobby for many people, a life profession for some. There are groups of tree hunters who strive to discover the mightiest specimens of individual tree species, in some countries even competing with rival groups to discover the biggest trees (www.treehunter.co.uk, www.dendromania.hu). These trees are measured and entered in databases of champion trees, tallest trees and similar.

American Forest Champion Trees National Register (www.americanforests.org) is one of the best-known databases, containing over 700 national champion trees. It was established 
back in 1940 and its impressive history inspired many other countries. In Europe, the Tree Register in Great Britain is one of the most important databases (www.treeregister.org). The Tree Register of the British Isles (T.R.O.B.I.) is a registered charity collecting and updating a database of champion trees. Since the 1980s champion trees registers have been established in most European countries, collecting numerous data and publishing some of them. For example, Mittchell et al. (1990), Jonson (2003) or Rodger et al. (2006) published papers on champion trees in Great Britain, as did Graaf (1991) in Holland or Baudoin et al. (1992) or Jacobs (2009) in Belgium. At present, champion trees in Germany are registered under the German Dendrological Society (DDG), with publications released by Kühn et al. (2005).

Information on Spanish champion and remarcable trees is provided by Lillo (1997) or later Lerena (2009) or Moya et Moya (2013). The latest publications related to champion trees come from France (Feterman, 2015) or Poland (Borkowski et al., 2016).

An increasing number of people all around the world have become interested in remarkable and magnificent trees in recent years. Accessible databases of champion trees are available even in Hongkong or New Zealand. For several years Europe has held the popular European Tree of the Year contest, which is a follow-up of national contests whose winners are nominated for the European competition. Furthermore, the European Champion Tree Forum (ECTF) was established in 2010. The ECTF is an organization of tree-lovers who take an interest in finding, measuring, documenting and publishing of remarkable, biggest, tallest, oldest "champion trees" in their respective countries as basis for wider activities to protect and preserve these trees. One is unthinkable without the other. The first meeting took place in Wespelaar, Belgium, in 2010, the last one in the Czech Republic in 2016 (www.championtrees.eu).

The development of the Internet has enabled many databases to go on-line, the international database Monumental Trees (www.monumentaltrees.com) being among the most comprehensive and best-known. This worldwide, publicly accessible database contains information on champion trees of many countries and continents. At present, it registers over 30,000 trees. Contrary to this, the paid database Monumental Trees of the World (www.goldencarers.com/monumental-trees-of-the-world) does not contain nearly as much information.

An increased interest in remarkable trees was registered on the territory of Czech lands as early as the turn of the $19^{\text {th }}$ and $20^{\text {th }}$ centuries. In 1899 Jan Evangelista Chadt-Ševětínský published the first survey of remarkable trees in Bohemia. A more detailed list of notable trees was published by the same author in 1908 in the popular and still existing magazine called Český lid (Czech People) under the title "Old and Memorable Trees in Bohemia, Moravia and Silesia", providing the description of 165 remarkable trees and 30 depictions. The emergence of the so-called beautifying societies (the Union of Czech Beautifying Societies in the Bohemian Kingdom was established in Prague on October 30 1904) falls into the same period. After the establishment of the Czechoslovak Republic it was transformed into the Union of Czechoslovak Societies for Beautification and Conservation of Homeland. Starting with 1920, the provision of paragraph 20 of the Allocations Act came into force to conserve remarkable trees. The relationship between people, forests and trees found its legislative basis.

The end of World War 2 marked the beginning of a "Survey of Memorable or Remarkable Trees, Tree Avenues and Forests" which was conducted by volunteers until 1956, using questionnaires of the State Institute of Cultural Heritage and Nature Conservation. Sadly, most of these records were lost. Act No. 40/1956 on State Nature Conservation allowed the designation of selected trees as protected nature monuments. However, their recording on the regional scale was not unified, bordering on confused at times. After the fall of the 
communist regime, a new Act No. 114/1992 Coll. on Nature and Landscape Protection has allowed the designation of selected remarkable trees as the so-called memorable trees based on an administrative decision in accordance with paragraph 46.

The Central Register of Nature Conservation, which is run by the Nature Conservation Agency of the Czech Republic, registered a total of 25,598 memorable trees in 2017, almost $80 \%$ of them in the form of solitary trees and the remaining $20 \%$ as tree avenues, tree groups and alleys. However, not only trees of extraordinary dimensions are designated as memorable. They may be remarkable for other reasons, their planting commemorating a special event or for being part of a legend. Out of the total number of solitary trees, 1,560 are lime trees and 1,180 oaks. Tree avenues also consist mainly of lime trees - they contain over 6,000 specimens and approximately 2,000 oaks. The remaining trees are mainly broadleaved, with a small percentage of conifers. All of them are state-protected, their legislative designation referring to them as "memorable trees".

The Vejda's Lime Tree (Vejdova lípa) in Pastviny in the Orlické Mts. is the mightiest champion - memorable tree in the country, its age estimated at over 700 years. Memorable trees are subject to frequent studies, such as those by Dundychová (1995), Kovaríík et al. (1996), Hrušková (1999, 2008, 2009) or Hrušková, Úradníček (2016), as well as Svoboda (1975), Donéeová, Janočko (2003), Taraba, Navrátilová (2005), Michálek (2006, 2008), Reš, Sůrová (2008), Kyzlík (2011) or Reš (2012). At present, records on memorable trees from 77 districts of the Czech Republic are available (www.cs.wikipedia.org).

Not all remarkable or champion trees are designated as memorable. In the landscape of the Czech Republic there is a number of mighty tree specimens of above-average size within their respective species (Maděra et al., 2000). Apart from the Central Register of Nature Conservation on memorable trees (http://drusop.nature.cz), there is also the database of Remarkable Trees of Forests of the Czech Republic, State Enterprise (www.lcr.cz) available. Another database which was run for several years is the database of Remarkable Trees of Military Forests and Farms of the Czech Republic, State Enterprise. At present, it provides on-line records only of memorable trees which grow on the company's land (www.vls.cz). The database of the Professor August Bayer Foundation is one of the oldest Czech databases. It lists the mightiest trees from the known databases, available publications and following their own measurements, partly as findings of the "Looking for Largest Trees" project launched by the Foundation in 1999 (Maděra \& Úradníček, 1999; 2000). Many champion trees grow in chateau parks and gardens, as shown by Hieke $(1984,1985)$ or Krejčiŕík et al. (2015). One of the first publications which also lists other champion trees than memorable trees comes from South Moravia, the Lower Morava Biosphere Reserve (Maděra et al., 2007).

The Czech Republic abounds in ancient castles, imposing chateaux and beautiful churches. However, champion trees which witnessed historical events in real time represent rare historical monuments as well. Compared with monuments created by people, these living natural monuments, very often state-protected memorable trees, have a major advantage on their side: they are alive. Their strength is simultaneously their weakness, as they are subject to the laws of life, ergo the law of decline. While a castle can be restored, a chapel rebuilt, living organisms disappear forever (Hrušková \& Úradníček, 2016). That is why we decided to list the monumental trees that grow in the Czech Republic.

Unfortunately, the Czech Republic does not have a single database of remarkable or champion trees. Many of the biggest specimens are already registered in the Monumental Trees database, yet a comprehensive database like the Hungarian Dendromania (www.dendromania.hu) or the Tree Register is still lacking. The aim of this paper is to 
Úradníček L., Šrámek M., Dreslerová J.: Checklist of champion trees in the Czech Republic

present the known data on monumental trees in an independent checklist as a basis for a future database.

\section{METHODS}

Trees are usually listed in databases based on their girth. In European countries, with the exception of Great Britain and Ireland, it is measured at the height of 1,3 metres above the ground, while in the two countries mentioned above it is $1,5 \mathrm{~m}$ above the ground. The measurement is taken perpendicular to the axis of the trunk. This is the method of measuring girth in the trees listed below. Height was measured by a Nikon Forestry Pro laser rangefinder. The trees were also localized (district, cadastre, possibly their GPS coordinates). The information recorded includes the year of measurement and the basic source of the data used.

\section{RESULTS AND DISCUSSION}

The table shows a list of trees in alphabetical order of their scientific names. Each species is represented by a single champion specimen based on its girth.

The following data sources (column 7) were used:

1 - Database of the Nature Conservation Agency of the Czech Republic (ÚSOP - memorable trees)

2 - Database of the Professor August Bayer Foundation

3 - Hrušková \& Úradníček, 2016 (List of Remarkable Champion Trees of the Czech

Republic)

4 - Krejčiřík et al., 2015 (Trees of the Lednice Chateau Park)

5 - Maděra et al., 2017 (Remarkable Trees and other Dendrological Points of Interest of the Lower Morava Biosphere Reserve)

6 - Businský \&Velebil, 2015 (Pines in the Czech Republic)

Table 1: Champion trees of the Czech Republic - checklist

\begin{tabular}{|l|c|c|c|c|c|c|c|}
\hline $\begin{array}{l}\text { Scientific } \\
\text { name }\end{array}$ & District & Cadastre & $\begin{array}{c}\text { Plot } \\
\text { number }\end{array}$ & $\begin{array}{c}\text { Tree } \\
\text { height } \\
(\mathbf{m})\end{array}$ & $\begin{array}{c}\text { Girth at } \\
\mathbf{1 . 3 ~ m} \\
(\mathbf{c m})\end{array}$ & Source & $\begin{array}{c}\text { Measured } \\
\text { (year) }\end{array}$ \\
\hline Abies alba & $\begin{array}{l}\text { Frýdek } \\
\text { Místek }\end{array}$ & Mionší & & 50 & 560 & 2 & 1999 \\
\hline Abies concolor & Liberec & Starý Harcov & 520 & 27 & 276 & 2 & 2008 \\
\hline Abies grandis & Blansko & Křtiny & $915 / 10$ & 37 & 395 & 2 & 2016 \\
\hline Abies homolepis & Šluknov & Šluknov & $1329 / 1$ & 23 & 198 & 1 & 2009 \\
\hline $\begin{array}{l}\text { Abies } \\
\text { nordmaniana }\end{array}$ & Blansko & Křtiny & $920 / 2$ & 31 & 227 & 2 & 2016 \\
\hline Abies procera & Děčín & Velký Šenov & $1711 / 2$ & 26 & 281 & 1 & 2009 \\
\hline Acer campestre & $\begin{array}{c}\text { Ustínad } \\
\text { Orlicí }\end{array}$ & Ústí nad Orlicí & $43 / 33$ & 21 & 500 & 1 & 2009 \\
\hline Acer platanoides & $\begin{array}{c}\text { Frýdek } \\
\text { Místek }\end{array}$ & $\begin{array}{c}\text { Krásná pod } \\
\text { Lysou horou }\end{array}$ & $1876 / 1$ & 19 & 460 & 1 & 2009 \\
\hline $\begin{array}{l}\text { Acer } \\
\text { pseudoplatanus }\end{array}$ & Třebíć & $\begin{array}{c}\text { Horní } \\
\text { Vilémovice }\end{array}$ & $197 / 2$ & 16 & 758 & 3 & 2010 \\
\hline $\begin{array}{l}\text { Acer } \\
\text { saccharinum }\end{array}$ & Děč́n & Jiř́́kov & $96 / 1$ & 25 & 502 & 1 & 2009 \\
\hline
\end{tabular}


Journal of Landscape Ecology (2017), Vol: 10 / No. 2

\begin{tabular}{|c|c|c|c|c|c|c|c|}
\hline $\begin{array}{l}\text { Aesculus } \\
\text { hippocastanum }\end{array}$ & Bruná & $\begin{array}{l}\text { Nová Ves u } \\
\text { Rýmařova }\end{array}$ & 96 & 28 & 589 & 1 & 2009 \\
\hline $\begin{array}{l}\text { Ailanthus } \\
\text { altissima }\end{array}$ & Brno-město & Chrlice & & 22 & 360 & 2 & 2000 \\
\hline Alnus glutinosa & Cheb & Beranovka & $627 / 3$ & 18 & 624 & 1 & 2014 \\
\hline Alnus incana & Nový Jičín & & & 25 & 140 & 2 & 2004 \\
\hline Betula nigra & Opava & Raduň & & 25 & 190 & 2 & 2002 \\
\hline Betula pendula & Šumperk & Šumperk & & 27 & 550 & 2 & 2005 \\
\hline Betula pubescens & Nový Jičín & Nový Jičín & & 22 & 180 & 2 & 2005 \\
\hline Carpinus betulus & Břeclav & Lanžhot & $3520 / 2$ & 16 & 463 & 2 & 2016 \\
\hline Carya ovata & Břeclav & Hlohovec & $1882 / 1$ & 15 & 157 & 2 & 2016 \\
\hline Castanea sativa & Chrudim & Nasavrky & $4 / 2$ & & 534 & 2 & 2016 \\
\hline $\begin{array}{l}\text { Catalpa } \\
\text { bignonioides }\end{array}$ & Hodonín & Strážnice & & 16 & 390 & 2 & 2003 \\
\hline Catalpa speciosa & Brno - město & Veveří & 1237 & 18 & 218 & 1 & 2009 \\
\hline Cedrus atlantica & $\begin{array}{l}\text { Hlavní město } \\
\text { Praha } \\
\end{array}$ & Vysočany & $1919 / 1$ & 20 & 253 & 1 & 2009 \\
\hline Cedrus libanini & $\begin{array}{l}\text { Praha - } \\
\text { západ }\end{array}$ & Černošice & $674 / 1$ & 22 & 340 & 1 & 2009 \\
\hline $\begin{array}{l}\text { Celtis } \\
\text { occidentalis }\end{array}$ & $\begin{array}{l}\text { Brno - } \\
\text { venkov }\end{array}$ & Střelice u Brna & $3441 / 16$ & 15 & 255 & 1 & 2015 \\
\hline Cerasus mahaleb & $\begin{array}{c}\text { Mladá } \\
\text { Boleslav }\end{array}$ & Nové Benátky & $2 / 1$ & 12 & 368 & 1 & 2009 \\
\hline $\begin{array}{l}\text { Cercidiphyllum } \\
\text { japonicum }\end{array}$ & Nový Jičín & $\begin{array}{c}\text { Frenštát pod } \\
\text { Radhoštěm }\end{array}$ & $442 / 1$ & 16 & 276 & 1 & 2011 \\
\hline Corylus colurna & Karviná & Český Těšín & $302 / 1$ & 22 & 357 & 1 & 2009 \\
\hline $\begin{array}{l}\text { Cryptomeria } \\
\text { japonica }\end{array}$ & Blansko & Křtiny & $915 / 10$ & 29 & 225 & 2 & 2016 \\
\hline $\begin{array}{l}\text { Cunninghamia } \\
\text { lanceolata }\end{array}$ & Brno - město & Veveří & $1022 / 1$ & 14 & 88 & 2 & 2016 \\
\hline Fagus sylvatica & $\begin{array}{c}\text { Rychnov nad } \\
\text { Kněžnou }\end{array}$ & $\begin{array}{c}\text { Pěčín u } \\
\text { Rychnova nad } \\
\text { Kněžnou } \\
\end{array}$ & $1834 / 1$ & 23 & 655 & 1 & 2009 \\
\hline $\begin{array}{l}\text { Fraxinus } \\
\text { angustifolia }\end{array}$ & Kroměříž & Litenčice & & 26 & 660 & 5 & 2006 \\
\hline $\begin{array}{l}\text { Fraxinus } \\
\text { excelsior }\end{array}$ & $\begin{array}{c}\text { Jindřichův } \\
\text { Hradec }\end{array}$ & $\begin{array}{l}\text { Budkov u } \\
\text { Stř́žzovic }\end{array}$ & 2 & 27 & 802 & 1 & 2012 \\
\hline Ginkgo biloba & Brno-město & Staré Brno & 8 & 25 & 446 & 2 & 2016 \\
\hline $\begin{array}{l}\text { Gleditsia } \\
\text { triacanthos }\end{array}$ & Břeclav & Lednice & 1863 & 29 & 372 & 2 & 2016 \\
\hline $\begin{array}{l}\text { Gymnocladus } \\
\text { dioicus }\end{array}$ & Nymburk & Lysá nad Labem & 178 & 16 & 286 & 1 & 2009 \\
\hline $\begin{array}{l}\text { Chamaecyparis } \\
\text { lawsoniana }\end{array}$ & Blansko & Křtiny & $920 / 2$ & 21 & 198 & 2 & 2016 \\
\hline $\begin{array}{l}\text { Chamaecyparis } \\
\text { nootkatensis }\end{array}$ & Olomouc & & & & 159 & 2 & 2000 \\
\hline $\begin{array}{l}\text { Chamaecyparis } \\
\text { obtusa }\end{array}$ & Blansko & Křtiny & $915 / 10$ & 15 & 117 & 2 & 2016 \\
\hline $\begin{array}{l}\text { Chamaecyparis } \\
\text { pisifera }\end{array}$ & Blansko & Křtiny & $915 / 10$ & 21 & 216 & 2 & 2016 \\
\hline Juglans nigra & Kroměříž & Kvasice & 152 & 25 & 672 & 1 & 2007 \\
\hline Juglans regia & Chrudim & Libkov & & 18 & 342 & 2 & 1999 \\
\hline $\begin{array}{l}\text { Juniperus } \\
\text { communis }\end{array}$ & Písek & Kučeř & 1843 & 8 & 135 & 1 & 2011 \\
\hline
\end{tabular}


Úradníček L., Šrámek M., Dreslerová J.: Checklist of champion trees in the Czech Republic

\begin{tabular}{|c|c|c|c|c|c|c|c|}
\hline $\begin{array}{l}\text { Juniperus } \\
\text { virginiana }\end{array}$ & Břeclav & Lednice & $5 / 4$ & 18 & 263 & 2 & 2016 \\
\hline Kalopanax pictus & $\begin{array}{c}\text { Jindřichův } \\
\text { Hradec }\end{array}$ & Nová Bystřice & 14 & 12 & 316 & 1 & 2016 \\
\hline Larix decidua & $\begin{array}{l}\text { Ústí nad } \\
\text { Labem }\end{array}$ & $\begin{array}{l}\text { Petrovice u } \\
\text { Chabařovic }\end{array}$ & 2185 & 28 & 683 & 1 & 2010 \\
\hline Larix kaempferi & Bruntál & Heřmanovice & 724 & 25 & 256 & 1 & 2009 \\
\hline $\begin{array}{l}\text { Liquidambar } \\
\text { orientalis }\end{array}$ & Brno - město & Veveří & $1022 / 1$ & 17 & 216 & 2 & 2016 \\
\hline $\begin{array}{l}\text { Liriodendron } \\
\text { tulipifera }\end{array}$ & Kroměříž & Kroměříž & $286 / 1$ & 37 & 493 & 1 & 2012 \\
\hline $\begin{array}{l}\text { Magnolia } \\
\text { acuminata }\end{array}$ & Děčín & $\begin{array}{c}\text { Kamenická Nová } \\
\text { Výska }\end{array}$ & 362 & 24 & 282 & 1 & 2016 \\
\hline Malus domestica & Přerov & Jezernice & & 10 & 220 & 2 & 2004 \\
\hline $\begin{array}{l}\text { Metasequoia } \\
\text { glyptrostroboides }\end{array}$ & Břeclav & Lednice & 1865 & 34 & 355 & 4 & 2014 \\
\hline Morus alba & Přerov & Olšovec & $1403 / 3$ & 11 & 318 & 1 & 2009 \\
\hline $\begin{array}{l}\text { Pawlovnia } \\
\text { tomentosa }\end{array}$ & Brno-město & Brno-město & & 17 & 380 & 2 & 1999 \\
\hline $\begin{array}{l}\text { Phellodendron } \\
\text { Amiense }\end{array}$ & Semily & Turnov & $1818 / 1$ & 18 & 423 & 1 & 2003 \\
\hline Picea abies & Sokolov & Šindelová & 446 & 36 & 505 & 1 & 2014 \\
\hline Picea pungens & Olomouc & Olomouc & & 34 & 215 & 2 & 2004 \\
\hline Pinus aristata & Prostějov & $\begin{array}{l}\text { Čechy pod } \\
\text { Kosířem }\end{array}$ & 153 & 14 & 123 & 6 & 2010 \\
\hline $\begin{array}{l}\text { Pinus armandi } \\
\text { Franch. }\end{array}$ & $\begin{array}{c}\text { Hlavní město } \\
\text { Praha }\end{array}$ & Zbraslav & 154 & 10 & 108 & 6 & 2010 \\
\hline Pinus cembra & Jeseník & Ostružná & 349 & 15 & 168 & 1 & 1981 \\
\hline Pinus coulteri & $\begin{array}{l}\text { Praha - } \\
\text { východ }\end{array}$ & $\begin{array}{l}\text { Kostelec nad } \\
\text { Černými lesy }\end{array}$ & $3109 / 1$ & 16 & 172 & 6 & 2010 \\
\hline Pinus fleklis & Brno & Černá Pole & $2 / 2$ & 20 & 152 & 6 & 2010 \\
\hline Pinus Jeffreyi & $\begin{array}{c}\text { Český } \\
\text { Krumlov }\end{array}$ & Pernek & 6 & 23 & 270 & 1 & 2010 \\
\hline Pinus nigra & Turnov & & & 40 & 497 & 2 & 2002 \\
\hline Pinus peисе & Sokolov & Kraslice & $5356 / 1$ & 27 & 275 & 1 & 2014 \\
\hline Pinus pinaster & \begin{tabular}{|c|}
$\begin{array}{c}\text { Hlavní město } \\
\text { Praha }\end{array}$ \\
\end{tabular} & Vyšehrad & $61 / 17$ & 11 & 130 & 6 & 2010 \\
\hline Pinus ponderosa & $\begin{array}{c}\text { Praha - } \\
\text { západ }\end{array}$ & Průhonice & & 40 & 375 & 6 & 2010 \\
\hline Pinus rigida & $\begin{array}{c}\text { Praha - } \\
\text { západ }\end{array}$ & Průhonice & & 32 & 213 & 6 & 2009 \\
\hline Pinus strobus & Blansko & Křetín & & 9 & 650 & 2 & 2004 \\
\hline Pinus sylvestris & Tachov & Lom u Tachova & 564 & 13 & 481 & 1 & 2009 \\
\hline $\begin{array}{l}\text { Pinus } \\
\text { wallichiana } \\
\end{array}$ & Šumperk & Šumperk & & 30 & 250 & 2 & 2004 \\
\hline $\begin{array}{l}\text { Platanus } \\
\text { orientalis }\end{array}$ & Nový Jičín & Kopřivnice & & 22 & 337 & 2 & 2002 \\
\hline $\begin{array}{l}\text { Platanus } \times \\
\text { acerifolia }\end{array}$ & Nový Jičín & $\begin{array}{c}\text { Bartošovice na } \\
\text { Moravě }\end{array}$ & $971 / 2$ & 36 & 842 & 2 & 2000 \\
\hline $\begin{array}{l}\text { Platycladus } \\
\text { orientalis }\end{array}$ & Břeclav & Lednice & & 26 & 190 & 2 & 2003 \\
\hline Populus alba & $\begin{array}{l}\text { Uherské } \\
\text { Hradiště }\end{array}$ & Suchá Loz & & 28 & 780 & 2 & 2000 \\
\hline Populus nigra & Nymburk & Nymburk & $390 / 5$ & 25 & 789 & 2 & 2016 \\
\hline Populus tremula & Znojmo & Lechovice & & & 430 & 2 & 2003 \\
\hline
\end{tabular}


Journal of Landscape Ecology (2017), Vol: 10 / No. 2

\begin{tabular}{|c|c|c|c|c|c|c|c|}
\hline $\begin{array}{l}\text { Populus } \times \\
\text { canadensis }\end{array}$ & Strakonice & Strakonice & & 15 & 580 & 2 & 2001 \\
\hline $\begin{array}{l}\text { Populus } \times \\
\text { canescens }\end{array}$ & $\begin{array}{l}\text { Uherské } \\
\text { Hradiště }\end{array}$ & Suchá Loz & $1206 / 2$ & 26 & 715 & 1 & 2010 \\
\hline Prunus avium & Trutnov & Hrádeček & $350 / 1$ & 28 & 423 & 1 & 2009 \\
\hline $\begin{array}{l}\text { Prunus } \\
\text { domestica }\end{array}$ & Hodonín & Mutěnice & & 8 & 142 & 2 & 2002 \\
\hline $\begin{array}{l}\text { Pseudotsuga } \\
\text { menziesii }\end{array}$ & Strakonice & $\begin{array}{c}\text { Čekanice u } \\
\text { Blatné }\end{array}$ & & 30 & 474 & 2 & 2001 \\
\hline Pyrus communis & Domažlice & Únějovice & 934 & 12 & 480 & 1 & 2009 \\
\hline Pyrus pyraster & Kroměříž & Cvrčovice & $100 / 2$ & 17 & 390 & 2 & 2004 \\
\hline Quercus cerris & Břeclav & Valtice & 5449 & 20 & 720 & 2 & 2005 \\
\hline Quercus frainetto & Brno-město & Černá Pole & $3857 / 1$ & 20 & 540 & 2 & 2008 \\
\hline $\begin{array}{l}\text { Quercus } \\
\text { macranthera }\end{array}$ & Nymburk & Mcely & $179 / 2$ & 25 & 452 & 1 & 2009 \\
\hline Quercus petraea & $\begin{array}{l}\text { Praha - } \\
\text { východ }\end{array}$ & Myšlín & $512 / 4$ & 18 & 700 & 1 & 2016 \\
\hline $\begin{array}{l}\text { Quercus } \\
\text { pubescens }\end{array}$ & Znojmo & Lechovice & $330 / 1$ & 16 & 647 & 2 & 2016 \\
\hline Quercus robur & Třebíč & $\begin{array}{l}\text { Náměšt nad } \\
\text { Oslavou }\end{array}$ & $135 / 1$ & 20 & 1030 & 3 & 2016 \\
\hline Quercus rubra & Pelhřimov & Proseč - Obořiště & $71 / 1$ & 25 & 660 & 1 & 2009 \\
\hline $\begin{array}{l}\text { Robinia } \\
\text { pseudoacacia }\end{array}$ & Hodonín & Strážnice & & 15 & 590 & 2 & 2005 \\
\hline Salix alba & Brno město & Bosonohy & 542 & 26 & 820 & 1 & 2010 \\
\hline Salix caprea & Šumperk & Rapotín & & 14 & 420 & 2 & 2005 \\
\hline Salix fragilis & Trutnov & Černý důl & 1128 & 14 & 540 & 1 & 2010 \\
\hline $\begin{array}{l}\text { Sciadopitys } \\
\text { verticillata }\end{array}$ & Blansko & Křtiny & $915 / 10$ & 11 & 90 & 2 & 2016 \\
\hline $\begin{array}{l}\text { Sequoiadendron } \\
\text { giganteum }\end{array}$ & $\begin{array}{l}\text { Uherské } \\
\text { Hradiště }\end{array}$ & Břestek & 2444 & 33 & 625 & 1 & 2010 \\
\hline Sorbus aria & Kladno & Kišice & 107 & 10 & 277 & 1 & 2012 \\
\hline Sorbus domestica & Hodonín & $\begin{array}{l}\text { Strážnice na } \\
\text { Moravě }\end{array}$ & 4295 & 17 & 483 & 2 & 2017 \\
\hline Sorbus torminalis & $\begin{array}{l}\text { Uherské } \\
\text { Hradiště }\end{array}$ & $\begin{array}{l}\text { Vápenice u } \\
\text { Starého } \\
\text { Hrozenkova }\end{array}$ & $7039 / 1$ & 18 & 380 & 1 & 2010 \\
\hline $\begin{array}{l}\text { Styphnolobium } \\
\text { japonicum }\end{array}$ & Břeclav & Lednice & $5 / 4$ & 20 & 581 & 2 & 2016 \\
\hline $\begin{array}{l}\text { Taxodium } \\
\text { distichum }\end{array}$ & Plzeň - jih & Dolní Lukavice & 27 & 19 & 399 & 1 & 2009 \\
\hline Taxus baccata & $\begin{array}{l}\text { Brno - } \\
\text { venkov }\end{array}$ & Pernštejn & 54 & 19 & 463 & 1 & 2014 \\
\hline $\begin{array}{l}\text { Thuja } \\
\text { occidentalis }\end{array}$ & Blansko & Křtiny & $915 / 10$ & 17 & 165 & 2 & 2016 \\
\hline Thuja plicata & Olomouc & Dalov & 112 & 28 & 383 & 1 & 2015 \\
\hline $\begin{array}{l}\text { Thujopsis } \\
\text { dolobrata }\end{array}$ & Blansko & Křtiny & $5 / 1$ & 12 & 116 & 2 & 2016 \\
\hline Tilia cordata & $\begin{array}{l}\text { Ústí nad } \\
\text { Labem }\end{array}$ & Český Bukov & $381 / 1$ & 14 & 925 & 1 & 2009 \\
\hline Tilia platyphyllos & $\begin{array}{l}\text { Ústí nad } \\
\text { Orlicí }\end{array}$ & $\begin{array}{c}\text { Pastviny u } \\
\text { Klášterce na } \\
\text { Orlicí }\end{array}$ & 499 & 16 & 1305 & 3 & 2015 \\
\hline Tilia tomentosa & Svitavy & $\begin{array}{c}\text { Svitavy } \\
\text { předměstí }\end{array}$ & $1916 / 41$ & 25 & 345 & 2 & 2000 \\
\hline
\end{tabular}


Úradníček L., Šrámek M., Dreslerová J.: Checklist of champion trees in the Czech Republic

\begin{tabular}{|l|c|c|c|c|c|c|c|}
$\begin{array}{l}\text { Torreya } \\
\text { californica }\end{array}$ & Břeclav & Lednice & $5 / 4$ & 12 & 220 & 5 & 2006 \\
\hline Torreya nucifera & Brno - město & Veveří & $1022 / 1$ & 18 & 212 & 2 & 2016 \\
\hline Tsuga canadensis & Děčín & Šluknov & $1343 / 1$ & 20 & 282 & 1 & 2009 \\
\hline $\begin{array}{l}\text { Tsuga } \\
\text { mertensiana }\end{array}$ & Děčín & Šluknov & $327 / 1$ & 14 & 97 & 1 & 2009 \\
\hline Ulmus glabra & $\begin{array}{c}\text { Frýdek } \\
\text { Místek }\end{array}$ & $\begin{array}{c}\text { Bukovec u } \\
\text { Jablunkova }\end{array}$ & 1350 & 38 & 730 & 1 & 2012 \\
\hline Ulmus laevis & Jeseník & Velká Kraš & $134 / 1$ & 23 & 658 & 1 & 2009 \\
\hline Ulmus minor & Vsetín & Liptál & 221 & 22 & 556 & 1 & 2007 \\
\hline Zelkova serrata & Břeclav & Hlohovec & $1882 / 1$ & 16 & 226 & 2 & 2014 \\
\hline
\end{tabular}

When comparing the champion trees of the Czech Republic with the databases of other European states, particularly that of Monumental Trees, it emerges that we are not far from coming first. The Czech champion tree, Vejda's Lime Tree in Pastviny (Tilia platyphyllos Scop.) measured $1,305 \mathrm{~cm}$ at girth in 2015. Compared with the largest specimen of this species, which grows in Germany, it falls short by $234 \mathrm{~cm}$, yet it comes third in European tables. The majestic lime tree in Telecí, which is called the Singing Lime Tree (Zpívající lípa) or Lukas's Lime Tree (Lukášova lípa) (Tilia platyphyllos, girth $1,170 \mathrm{~cm}$ ), is one of the most beautiful lime trees in Europe. It stands next to a traditional house in the highlands style and is one of the very few individual trees in Europe that ever made it on a postage stamp.

The champion oak of the Czech Republic is Žižka's Oak in Náměšt' nad Oslavou (Quercus robur L., girth $1,030 \mathrm{~cm}$ in 2017). It is interesting to note that this remarkable tree was not artificially planted but is a remnant of an original oak forest. Legend has it that the famous commander Žižka set camp under the oak when the Hussite army laid siege to the local castle and that he even tied his horse to it. Many of the cavities of the trunk and branches are covered with elaborate shingle roofs (Hrušková \& Úradníček, 2016). When compared with European champion oaks, it may be no match for oaks in Sweden (girth $1,510 \mathrm{~cm}$ ) or Denmark (girth 1,396 cm) but it can easily compete with champion oaks in Poland - "Dab Chrobry" - 1,004 cm (Zarzinski et al., 2016), Belgium - "Gros Chêne de Liernu" - girth $1,024 \mathrm{~cm}$ (Jacobs, 2009) or Lithuania (monumentaltrees.com).

A sycamore maple (Acer pseudoplatanus L.), known as the Large Maple in Věstoňovice, with its girth of $758 \mathrm{~cm}$ came sixth in the country rating according to www.monumentaltrees.com. It is our largest maple and probably the oldest as well. Records of this remarkable maple tree can be found in expert publications as early as the 19th century (Chadt-Ševětínský, 1899). Over the centuries its trunk has become a living artefact. The hollow trunk is covered by openings resulting from broken-off crown branches, while smaller branches are still alive, covered in leaves. Although it cannot compare with the largest European specimens, e.g. the Italian maple in Pizzo Lipo (girth 1,028 cm) or the German champion maple in Walberg (girth $900 \mathrm{~cm}$ ), its appearance and a very thin trunk shell walls make it unique within the Czech Republic.

The Adamec Service Tree (Sorbus domestica L.), with its girth of $483 \mathrm{~cm}$ in 2017, ranks high in European lists. This champion tree is probably the oldest in the country, growing on the Žerotín Hill in Radějov by Strážnice. The estimated age of this tree with a 20-metre crown spread is 400 years. This useful tree is probably a native fruit tree species whose history can be studied in the Service Tree Museum in Tvarožná Lhota (Hrušková \& Úradníček, 2016). Only a service tree in Slovakia, "Modranská oskoruše", with its girth of $500 \mathrm{~cm}$ is slightly bigger and beats this Czech champion tree to an honorary second place.

The hedge maple (Acer campestre L.), girth $500 \mathrm{~cm}$, which grows in Ústí nad Orlicí, is also the second largest European maple. A bigger specimen can only be found in the Dobrzyca 
park, Poland. According to Zarziński et al. (2016), the Polish champion tree measured only $460 \mathrm{~cm}$ at girth in 2011 , yet according to www.monumentaltrees.com it reached $516 \mathrm{~cm}$ at repeated measuring, which made it the European champion. Our maple was last measured in 2009 and it is possible that at present it has a bigger girth than the listed European champion maple.

According to www.monumentaltrees.com the wild pear tree (Pyrus pyraster (L.) Burgsd.) which grows in Kroměříž and whose girth is $390 \mathrm{~cm}$ is the third biggest in Europe. It will probably not grow bigger than the European champion tree from Poland, which grew to $560 \mathrm{~cm}$ in girth in Tarnowskie Góry.

A monumental pubescent oak (Quercus pubescens L.) which is living its last days in the Lechovice chateau park has a girth of $647 \mathrm{~cm}$. In the ranking of countries where trees are measured and the readings are listed on-line it comes fourth. It may not be an equal match to trees growing in warmer countries, such as Spain (girth $821 \mathrm{~cm})$, Italy $(723 \mathrm{~cm})$ or Croatia $(660 \mathrm{~cm})$, but it is an unexpectedly monumental tree for the Central European conditions.

As for conifers, we should note the Petrovice larch (Larix decidua Mill.) which measured $683 \mathrm{~cm}$ in girth in 2010. This ranks the Czech Republic third in Europe, surpassed only by Switzerland (girth $1,120 \mathrm{~cm}$ ) and Italy (girth $891 \mathrm{~cm}$ ) according to www.monumentaltrees.com.

We will conclude the list with a European champion tree according to www.monumentaltrees.com, the wych elm tree (Ulmus glabra Huds.) and its girth of $726 \mathrm{~cm}$ measured in 2012. This elm in Bukovec is called the "Border Guard" and is the largest elm in the Czech Republic. Its mighty trunk has strong root swellings, it branches at the height of eight metres and its crown is 35 metres high. It grows at the end of the village, a kilometre from the Czech-Polish border on the left bank of the Olše River, hence the name Border Guard. It is particularly valuable as it is the last large elm of the original six in our country which has survived the epidemic of Dutch elm disease. This elm is healthy, having received arborist treatment in 1996, when its cavity was roofed. Its estimated age is 350-500 years (Hrušková \& Úradníček, 2016). This specimen is a mere $3 \mathrm{~cm}$ larger in girth than the wych elm on the Brahan Estate in Great Britain which reached $723 \mathrm{~cm}$ in girth in 2013. However, the question remains whether the British elm's girth measured at $1.3 \mathrm{~m}$ above ground would not be bigger. The Tree Register database lists an even more monumental elm, the "Bishop Burton" with a girth of $928 \mathrm{~cm}$. However, the entry dates from 2011 and it is unclear whether the tree is still alive, as www.monumentaltrees.com would probably have listed it otherwise.

Apart from trees, there is an interesting specimen of the only native evergreen climber, the common ivy (Hedera helix L.), whose girth measured $97 \mathrm{~cm}$ in 2015. This specimen growing in South Moravia in the Pavlovské Hills is probably the largest in Europe. Its girth exceeds the Belgian champion ivy by $2 \mathrm{~cm}$ (girth $95 \mathrm{~cm}$, measured in 2012) as well as the Slovak champion (girth $90 \mathrm{~cm}$ ) - see www.monumentaltrees.com.

There are many other promising trees in the Czech Republic which have the potential of reaching monumental dimensions. But whether they get the possibility to achieve their full potential and reach an advanced age will depend primarily on people and their relationship to trees.

\section{CONCLUSION}

Interest in trees in the Czech Republic has been triggered by the documentary series "Memory of Trees" produced by the Czech Television, as well as many publications representing remarkable trees mostly on a regional level. Moreover, in 2000 the Tree of the Year contest was launched as well. However, the making of a central database of remarkable 
trees of the Czech Republic will still require considerable effort. Firstly, an independent portal (championtrees.cz) will need to be created and known, albeit not yet published data will have to be entered in it. The groundwork has been done - the table shown above and the remarkable trees database run by the Professor August Bayer Foundation. What remains is to expand and complement the data in the shortest possible time.

\section{ACKNOWLEDGMENTS:}

This work was supported by the Professor August Bayer Endowment Fund.

\section{REFERENCES}

Baudouin, J. C., De Spoelberch, Ph. \& Van Meulder, J. (1992). Bomen in Belgie. Dendrologische inventaris 1987-1992. Spoelberch-Artois Stichting, 511 pp.

Businský, R. \& Velebil, J. (2011). Borovice v České republice. Výsledky dlouhodobého hodnoceni rodu Pinus L. v kultuře v České republice. VÚKOZ, Průhonice, $180 \mathrm{~s}$.

Borkowski, B., Tomusiak, R. \& Zarzyński, P. (2016). Drzewa Polski. Warszawa. Wydawnictwo Naukowe PWN.

Dundychová, I. (1995). Památné stromy okresů Havličkưv Brod, Pelhřimov a Jihlava. Havlíčkův Brod, ČÚOP.

Feterman, G. (2015). Arbre et patrimoine de France. Plaissan. Museo Éditions.

Graaf, G.(1991). Monumentale Bomen in Nederland. Boom / Bomenstichting.

Hieke, K. (1984). České zámecké parky a jejich dřeviny. Praha, SZN.

Hieke, K. (1985). Moravské zámecké parky a jejich dřeviny. Praha, SZN.

Hrušková, M. (1999). Stromy pamatují. Manuskript. Praha.

Hrušková, M. (2008). Za památnými stromy Vysočiny. Beroun. Nakladatelství MH Beroun.

Hrušková, M. (2009). Za památnými stromy Vysočiny II. Beroun. Nakladatelství MH Beroun.

Hrušková, M. \& Úradníček, L. (2016). List of remarcable champion trees of the Czech Republic. Manuscript, Nadační fond prof. Augusta Bayera, Brno, 27pp.

Chadt-Ševětínský, J. E. (1899). Staré, památné a historické stromy vzemích Česko-Slovenských. Časopis Klubu českých turistů, 11: 50-51.

Chadt-Ševětínský, J. E. (1908). Staré a památné stromy v Čechách, na Moravě a ve Slezsku. Český lid 17, zvl. otisk.

Jacobs, R. (2009). Trees of Belgium Revisited. Belgium. Schoten. BAI Publisher.

Johnson, O., (2003). Champion trees of Britain and Ireland. The tree register handbook. University of Chicago Press, Chicago.

Kovařík, V., Pešout, P. \& Zelený, V. (1996). Zámecké parky a památné stromy Podblanicka. Vlaším, ČSOP Vlašim.

Krejčiř́ik, P., Pejchal, M., Šimek, P., Bulíř, P. \& Pavlačka, R. (2015). Dřeviny zámeckého parku v Lednici. Brno: Mendelova univerzita v Brně, 2015. ISBN 978-80-7509-356-1.

Kühn, U., Kühn, S. \& Ullrich, B. (2005). Bäume die Geschichten erzählen. München, BLV Buchen Verlag GmbH \& Co. KG. 
Kyzlík, P (2011). Jilmy památné a významné. In. Jilm: dřevina roku 2011: sborník referátů konference 20. 4. 2011, Kostelec nad Černými lesy, zámek. Vyd. 1. V Praze: Česká lesnická společnost, Česká zemědělská univerzita, $59 \mathrm{~s}$

Lerena, S. D. (2009). Gigantes y ancianos de los bosques españoles. Bosques Madrid. Sin Fronteras.

Lillo, A. L. (1997). Árboles Monumentales de Madrid. Madrid. Lunwerg Editores.

Maděra, P., Buček, A., Štykar J., Čermák, P., Hrubý, Z., Klečka, J., Král. K. \& Vojtek, M. (2000). Databank of important tree species in the Odra river watershed riparian zone of streams. Čas. Slezs. Muz. Opava (serie A), 49: 239-246, 2000

Maděra, P, Pejchal, M., Úradníček, L., Krejčiřík, P., Dreslerová, J., Klimánek, M., Mikita, T., Lička, D \& Čupa, P. (2007): Remarcable Trees and other Dendrological Points of Interest the Lower Morava Biosphere Reserve. BR Dolní Morava o.p.s., 73 pp.

Maděra, P. \& Úradníček, L. (2000). Pomozte nám hledat největší stromy. Lesnická práce, 79,1

Maděra, P. \& Úradníček, L. (1999). Utajený prales na soutoku Moravy a Dyje aneb „Hledáme největší stromy“, Veronica roč. 13, č. 5 Brno, p. 23-24

Michálek, J. (2006). Památné stromy Plzeňského kraje. Plzeň, Krajský úřad Plzeňského kraje.

Michálek, J. (2008). Památné stromy Karlovarského kraje. Sokolov, KMKK-Muzeum Sokolov.

Mitchell, A. F., Hallett, V. E., \& White, J. E. (1990). Champion trees in the British Isles. Edinburgh. Forestry Commission.

Moya, B. \& Moya J. (2013). Monumental trees and mature forests Threatened in the Mediterranean landscape. Valencia. IMELSA.

Pakenham, T. (2002). Remarkable trees of the world. London, Weidenfeld \& Nicolson.

Reš, B., (2012). Ochrana památných stromů. In: Machar I., Drobilová L. (eds) Ochrana prírody a krajiny v ČR I-II (576-587). Univerzita Palackého v Olomouci.

Reš, B. \& Sůrová, B. (2008). Památné stromy. AOPK ČR, Praha.

Rodger, D., Stokes, J. \& Ogilvie, J. (2006). Heritage trees of Scotland. London, Forestry Commisission Scotland.

Svoboda, A. M. (1975). Staré stromy - památníky přírody. Živa 23: 147 (in czech).

Taraba, R. \& Navrátilová, M. (2005). Památné stromy města Brna. Brno, MGMB.

Żukowski, A. (2006). Sławne drzewa województwa śląskiego. Stare Babice. Agencja Reklamowo-Wydawnicza" Vectra".

\section{ELECTRONICAL SOURCES (WWW)}

https://cs.wikipedia.org/wiki/Kategorie:Pam\%C3\%A1tn\%C3\%A9_stromy_v_\%C4\%8Cesk u_podle_okres\%C5\% AF

http://dendromania.hu/index.php

http://drusop.nature.cz/ost/chrobjekty/pstromy/

https://lesycr.cz/pece-o-les/ochrana-prirody-u-lcr/vyznamne-stromy/

https://www.vls.cz/cs/pro-verejnost/pamatne-stromy

http://www.championtrees.eu/ 
Úradníček L., Šrámek M., Dreslerová J.: Checklist of champion trees in the Czech Republic

http://www.treeregister.org/

http://www.treehunter.co.uk/

http://www.americanforests.org/explore-forests/americas-biggest-trees/champion-trees-nati onal-register/

http://www.monumentaltrees.com/en/europe - Monumental trees in Europe

https://www.monumentaltrees.com/en/ - Monumental trees

https://www.goldencarers.com/monumental-trees-of-the-world/4648/ - Monumental Trees Of The World. 